\title{
Symmetry origin of the phase transitions and phase separation in manganites at low doping
}

\author{
Fan Zhong and Z. D. Wang* \\ Department of Physics, University of Hong Kong, Hong Kong, People's Republic of China
}

(Received 16 February 1999)

\begin{abstract}
We analyze the symmetry changes of paramagnetic to $A$-type antiferromagnetic and to ferromagnetic phase transitions in undoped and moderately doped $\mathrm{LaMnO}_{3}$, respectively. We show that in orthorhombic-distorted perovskite manganites the phase separation at low doping is associated with the noncollinear nature of the magnetic orders permitted by symmetry. A simple model for the competition between the two phase transitions is put forward within the framework of the Landau theory of phase transitions. [S0163-1829(99)03038-6]
\end{abstract}

The discovery of "colossal" magnetoresistance (CMR) has stimulated a renaissance of interest in doped rare-earth manganese oxides because of their promising practical applications and their similarity to cuprate superconductors. ${ }^{1,2} \mathrm{Al}-$ though great efforts have been devoted to this system, the various phase transitions occurring under doping are still not fully understood as a result of the complex interplay among magnetic, charge, orbital, and structural orders. The present lack of a precise command of strong correlations makes difficult discriminating models based on, for example, pure double-exchange, Jahn-Teller, and doping variants. ${ }^{3}$ Therefore, it is desirable to investigate general properties such as the symmetry of the system that are feasible and meanwhile informative enough both to impose rigorous general restrictions and to shed light on microscopic theories.

Perovskite-structured $\mathrm{LaMnO}_{3}$ is believed to be a JahnTeller distorted orthorhombic structure with a crystallographic space group $\operatorname{Pnma}\left(D_{2 h}^{16}\right)$ at room temperature. ${ }^{4}$ Below $T_{N} \sim 140 \mathrm{~K}$, it undergoes a magnetic transition from a paramagnetic (PM) to an $A$-type antiferromagnetic (AFM) phase in which ferromagnetic (FM) layers are coupled antiferromagnetically, different from the usual AFM couplings along all nearest neighbors ( $G$ type), while its lattice remains unaltered. ${ }^{1}$ The $\mathrm{Mn}^{3+}\left(d^{4}\right)$ ion is believed to be in the $t_{2 g}^{3} e_{g}^{1}$ high-spin state, and strong on-site correlations render the compound insulating in both magnetic phases. Upon doping of divalent ions, some Mn ions lose their Jahn-Teller active $e_{g}^{1}$ electrons, leaving much smaller $\mathrm{Mn}^{4+}$ ions with mobile holes, a sufficient amount of which may make the lowtemperature phase metallic and ferromagnetic via a double exchange, ${ }^{5}$ a superexchange interaction between localized $t_{2 g}$ spins which is facilitated by an external magnetic field and hence follows the so-called colossal magnetoresistance. In addition to this magnetotransport behavior, many unusual phenomena show up such as the magnetostructural transition, charge and orbital orders, and their stability to external influences such as magnetic field, pressure, x-ray, electric field, and light irradiations. ${ }^{6}$ A particular issue that poses a great challenge to theorists, ${ }^{7}$ besides the mechanism of CMR itself, is the tendency of the system to phase separate not only at high doping through a first-order FM to chargeordered AFM transition, ${ }^{8}$ but also at low doping. ${ }^{1,9,10} \mathrm{We}$ shall show below by symmetry analysis that both the PM to AFM and the PM to FM transitions are induced by the same irreducible corepresentation, which, among others, permits a common FM component for both the AFM and FM phases. The competition between these two phases upon doping leads to an electronic phase separation at low doping when combined with the microscopic mechanism of double exchange.

First we analyze the symmetry change of the PM to AFM phase transition in undoped $\mathrm{LaMnO}_{3}$. Since the crystallographic space group remains unaltered during the magnetic phase transition, this transition must be associated with a one-dimensional irreducible corepresentation of the magnetic group at the center $(\mathbf{k}=0)$ of the orthorhombic Brillouin zone. As a result, the PM to AFM transition is governed by a single order parameter that acquires a nonzero value representing the staggered magnetization below $T_{N}$.

The magnetic group of the PM phase contains the timereversal operation $\mathcal{R}$ itself as one of its elements and so is the gray group $P n m a 1^{\prime}$, a direct product group of Pnma, and the group $\{E, \mathcal{R}\}$ with $E$ being the identity operation. ${ }^{11}$ All its irreducible corepresentations (ICR's) are thus multiplied into a doubled set of even and odd groups. Only the odd representations are relevant as $\mathcal{R}$ reverses the direction of a spin, and so the transition from a PM state can be described simply by the axial vector basis functions of the irreducible representations of the space group Pnma. ${ }^{12}$ Designating a space group element by $\left\{R \mid \mathbf{t}_{R}+\mathbf{t}\right\}$, where $R$ represents a proper or improper rotation, $\mathbf{t}_{R}$ a nonprimitive (fractional) translation associated with $R$, and $\mathbf{t}$ a primitive translation, the eight coset representatives of Pnma with respect to the subgroup of pure translations $\{E \mid \mathbf{t}\}$ are $^{13}$

\begin{tabular}{|c|c|c|c|c|c|c|c|}
\hline$h_{1}$ & $h_{2}$ & $h_{3}$ & $h_{4}$ & $h_{25}$ & $h_{26}$ & $h_{27}$ & $h_{28}$ \\
\hline$\{E \mid 000\}$ & $\left\{U_{x} \mid \frac{1}{2} \frac{1}{2} \frac{1}{2}\right\}$ & $\left\{U_{y} \mid 0 \frac{1}{2} 0\right\}$ & $\left\{U_{z} \mid \frac{1}{2} 0 \frac{1}{2}\right\}$ & $\{I \mid 000\}$ & $\left\{\sigma_{x} \mid \frac{1}{2} \frac{1}{2} \frac{1}{2}\right\}$ & $\left\{\sigma_{y} \mid 0 \frac{1}{2} 0\right\}$ & $\left\{\sigma_{z} \mid \frac{1}{2} 0 \frac{1}{2}\right\}$ \\
\hline
\end{tabular}


where $I$ denotes an inversion, $U_{x}$ a rotation by $\pi$ about the $x$ axis, $\sigma_{x}$ a reflection about the plane perpendicular to $x$, etc., and the $h_{i}$ 's are Kovalev's symbols. Then according to the character table of the point group $D_{2 h}$, Table I, the magnetic symmetries of the phases that arise from the corresponding irreducible representations can be determined as follows:

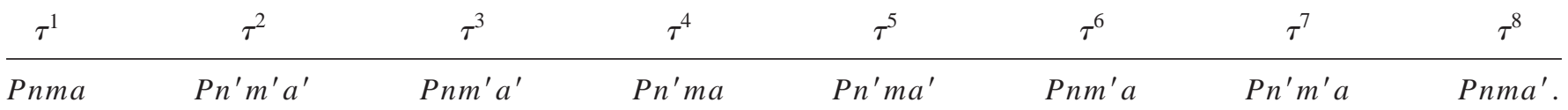

Here the primes indicate the symmetry elements that are associated with $\mathcal{R}$ in the respective magnetic groups; for example, all the reflection planes of $\tau^{2}$ must combine with $\mathcal{R}$ to give $P n^{\prime} m^{\prime} a^{\prime}$ since all their corresponding characters are $-1 .^{14}$

Next we determine the nature of the magnetic order below $T_{N}$. The elementary unit cell of $\mathrm{LaMnO}_{3}$ contains four formula units with $\mathrm{Mn}^{3+}$ ions located at the $4 a$ sites 1(000), $2\left(\frac{1}{2} 0 \frac{1}{2}\right), 3\left(0 \frac{1}{2} 0\right)$, and $4\left(\frac{1}{2} \frac{1}{2} \frac{1}{2}\right)$ (see Fig. 1). ${ }^{15}$ Associating each ion with a magnetic moment $\boldsymbol{\mu}$, one can find their transformations by $h_{i}$ 's as shown in Table II. Let

$$
\begin{aligned}
& \mathbf{M}=\boldsymbol{\mu}_{1}+\boldsymbol{\mu}_{2}+\boldsymbol{\mu}_{3}+\boldsymbol{\mu}_{4}, \\
& \mathbf{L}_{1}=\boldsymbol{\mu}_{1}-\boldsymbol{\mu}_{2}+\boldsymbol{\mu}_{3}-\boldsymbol{\mu}_{4}, \\
& \mathbf{L}_{2}=\boldsymbol{\mu}_{1}+\boldsymbol{\mu}_{2}-\boldsymbol{\mu}_{3}-\boldsymbol{\mu}_{4}, \\
& \mathbf{L}_{3}=\boldsymbol{\mu}_{1}-\boldsymbol{\mu}_{2}-\boldsymbol{\mu}_{3}+\boldsymbol{\mu}_{4},
\end{aligned}
$$

which represent, respectively, the total magnetization and three possible AFM collinear orders of $C, A$, and $G$ types. ${ }^{2,14,12}$ Then, according to Table II, the transformation properties of $\mathbf{M}$ and $\mathbf{L}_{i}$ 's can be derived. Noting that both $U_{x}$ and $\sigma_{x}$ change the sign of the $y$ and $z$ components of an axial vector, one deduces further the transformation properties of their respective components, from which those components that form the bases of the ICR's of Pnma $1^{\prime}$ at $\mathbf{k}=0$ can be found to be

\begin{tabular}{cl} 
ICR & Bases \\
\hline$\tau^{1}$ & $L_{3 x}, L_{1 y}, L_{2 z}$ \\
$\tau^{3}$ & $M_{x}, L_{2 y}, L_{1 z}$ \\
$\tau^{5}$ & $L_{2 x}, M_{y}, L_{3 z}$ \\
$\tau^{7}$ & $L_{1 x}, L_{3 y}, M_{z}$.
\end{tabular}

TABLE I. Characters of the irreducible representations of $P n m a$ at $k=0$.

\begin{tabular}{rrrrrrrrr}
\hline \hline & $h_{1}$ & $h_{2}$ & $h_{3}$ & $h_{4}$ & $h_{25}$ & $h_{26}$ & $h_{27}$ & $h_{28}$ \\
\hline$\tau^{1}$ & 1 & 1 & 1 & 1 & 1 & 1 & 1 & 1 \\
$\tau^{2}$ & 1 & 1 & 1 & 1 & -1 & -1 & -1 & -1 \\
$\tau^{3}$ & 1 & 1 & -1 & -1 & 1 & 1 & -1 & -1 \\
$\tau^{4}$ & 1 & 1 & -1 & -1 & -1 & -1 & 1 & 1 \\
$\tau^{5}$ & 1 & -1 & 1 & -1 & 1 & -1 & 1 & -1 \\
$\tau^{6}$ & 1 & -1 & 1 & -1 & -1 & 1 & -1 & 1 \\
$\tau^{7}$ & 1 & -1 & -1 & 1 & 1 & -1 & -1 & 1 \\
$\tau^{8}$ & 1 & -1 & -1 & 1 & -1 & 1 & 1 & -1 \\
\hline \hline
\end{tabular}

That is, $L_{2 x}$, for instance, transforms according to the representation $\tau^{5}$, and so do $M_{y}$ and $L_{1 z}$. Accordingly, it is straightforward to construct the Landau free energy that is an invariant:

$$
\begin{aligned}
F= & F_{0}+\sum_{i=1}^{3} \frac{a_{i}}{2} \mathbf{L}_{i}^{2}+\frac{c}{2} \mathbf{M}^{2}+\sum_{i=1}^{3} \frac{b_{i}}{4} \mathbf{L}_{i}^{4}+\frac{d}{4} \mathbf{M}^{4} \\
& +\frac{1}{2} \sum_{\alpha=x, y, z}\left(\sum_{i=1}^{3} \nu_{i \alpha} L_{i \alpha}^{2}+\beta_{\alpha} M_{\alpha}^{2}\right)+\gamma_{1} L_{3 x} L_{1 y} \\
& +\gamma_{2} L_{3 x} L_{2 z}+\gamma_{3} L_{1 y} L_{2 z}+\gamma_{4} M_{x} L_{2 y}+\gamma_{5} M_{x} L_{1 z} \\
& +\gamma_{6} L_{2 y} L_{1 z}+\gamma_{7} L_{2 x} M_{y}+\gamma_{8} L_{2 x} L_{3 z}+\gamma_{9} M_{y} L_{3 z} \\
& +\gamma_{10} L_{1 x} L_{3 y}+\gamma_{11} L_{1 x} M_{z}+\gamma_{12} L_{3 y} M_{z} .
\end{aligned}
$$

We have expanded the exchange contributions (first line) to the fourth order and the magnetic anisotropic energies (last four lines) to the second order because of their relatively smaller magnitude. Among all the coefficients, $b_{i}$ and $d$ are positive for stability, and $\gamma_{i}, \nu_{i \alpha}$, and $\beta_{\alpha}$ are small constants from relativistic effects. ${ }^{12,16}$ By ignoring the anisotropic contributions, it is readily seen that Eq. (4) may yield a FM or AFM phase of $G, A$, or $C$ type depending on the coefficients $a_{i}$ and $c$.

Experimentally, it has been observed that the magnetic structure of $\mathrm{LaMnO}_{3}$ is $A$-type AFM order with the magnetic moments directing primarily along $x$ axis. ${ }^{1,17}$ As a result, the magnetic transition is described by a nonzero $L_{2 x}$ below $T_{N}$. So $a_{2}$ should become negative first among $a_{i}$ and $c$ upon cooling. Retaining only those terms in Eq. (4) that contain the components pertinent to $\mathbf{L}_{2 x}$, we have

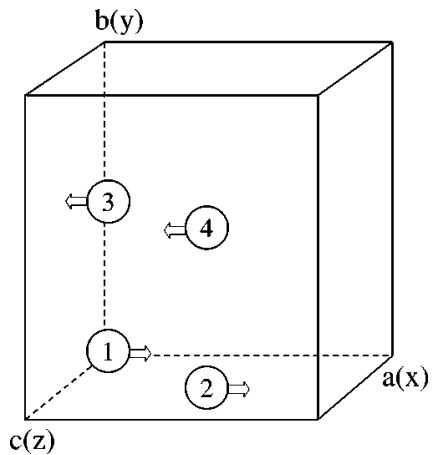

FIG. 1. Elementary unit cell containing four Mn ions with magnetic moments shown schematically by arrows. 
TABLE II. Transformation table of the magnetic moments.

\begin{tabular}{lllllllll}
\hline \hline & $h_{1}$ & $h_{2}$ & $h_{3}$ & $h_{4}$ & $h_{25}$ & $h_{26}$ & $h_{27}$ & $h_{28}$ \\
\hline $\boldsymbol{\mu}_{1}$ & $\boldsymbol{\mu}_{1}$ & $\boldsymbol{\mu}_{4}$ & $\boldsymbol{\mu}_{3}$ & $\boldsymbol{\mu}_{2}$ & $\boldsymbol{\mu}_{1}$ & $\boldsymbol{\mu}_{4}$ & $\boldsymbol{\mu}_{3}$ & $\boldsymbol{\mu}_{2}$ \\
$\boldsymbol{\mu}_{2}$ & $\boldsymbol{\mu}_{2}$ & $\boldsymbol{\mu}_{3}$ & $\boldsymbol{\mu}_{4}$ & $\boldsymbol{\mu}_{1}$ & $\boldsymbol{\mu}_{2}$ & $\boldsymbol{\mu}_{3}$ & $\boldsymbol{\mu}_{4}$ & $\boldsymbol{\mu}_{1}$ \\
$\boldsymbol{\mu}_{3}$ & $\boldsymbol{\mu}_{3}$ & $\boldsymbol{\mu}_{2}$ & $\boldsymbol{\mu}_{1}$ & $\boldsymbol{\mu}_{4}$ & $\boldsymbol{\mu}_{3}$ & $\boldsymbol{\mu}_{2}$ & $\boldsymbol{\mu}_{1}$ & $\boldsymbol{\mu}_{4}$ \\
$\boldsymbol{\mu}_{4}$ & $\boldsymbol{\mu}_{4}$ & $\boldsymbol{\mu}_{1}$ & $\boldsymbol{\mu}_{2}$ & $\boldsymbol{\mu}_{3}$ & $\boldsymbol{\mu}_{4}$ & $\boldsymbol{\mu}_{1}$ & $\boldsymbol{\mu}_{2}$ & $\boldsymbol{\mu}_{3}$ \\
\hline \hline
\end{tabular}

$$
\begin{aligned}
F^{\prime}= & \frac{a_{2}}{2} L_{2 x}^{2}+\frac{a_{3}}{2} L_{3 z}^{2}+\frac{b_{2}}{4} L_{2 x}^{4}+\frac{\nu_{2 x}}{2} L_{2 x}^{2}+\frac{c}{2} M_{y}^{2} \\
& +\frac{1}{2} \beta_{y} M_{y}^{2}+\gamma_{7} L_{2 x} M_{y}+\gamma_{8} L_{2 x} L_{3 z},
\end{aligned}
$$

with the solution

$$
\begin{gathered}
L_{2 x}^{2}=-\frac{1}{b_{2}}\left(a_{2}+\nu_{2 x}-\frac{\gamma_{7}^{2}}{c+\beta_{y}}-\frac{\gamma_{8}^{2}}{a_{3}}\right) \approx-\frac{a_{2}}{b_{2}}, \\
M_{y}=-\frac{\gamma_{7}}{c+\beta_{y}} L_{2 x} \approx-\frac{\gamma_{7}}{c} \sqrt{-\frac{a_{2}}{b_{2}}}, \\
L_{3 z}=-\frac{\gamma_{8}}{a_{3}} L_{2 x} \approx-\frac{\gamma_{8}}{a_{3}} \sqrt{-\frac{a_{2}}{b_{2}}},
\end{gathered}
$$

which minimizes $F^{\prime}$, where the last approximate equalities in each line neglect those terms that are orders of magnitude smaller.

Equations (6) correspond to an $A$-type AFM order with the magnetic moments directing primarily along the $\pm x$ axis in alternative $\mathrm{Mn}-\mathrm{O}$ layers perpendicular to the $y$ axis. Meanwhile, all the moments tilt slightly along both the $y$ and $z$ axes, giving rise to a weak FM and a weak $G$-type AFM order, respectively, in those two directions. The solution is associated with the irreducible corepresentations $\tau^{5}$, and the magnetic group of the asymmetry phase is thus $P n^{\prime} m a^{\prime}$ from the lists Eqs. (3) and (1). Actually, the orientation of the magnetic moments can also be obtained directly from the irreducible representation, except their relative magnitude. In other words, all three types of order along their respective directions are simultaneously allowed by the symmetry. So from the symmetry point of view, a canted phase is allowed. A weak ferromagnetic component along $y$ has been inferred and observed in experiments. ${ }^{17}$ The magnetic structure obtained also agrees with the results of local-spin-densityapproximation calculations. ${ }^{18}$ We note in passing that as the magnetic anisotropic energies arise from the relativistic spinorbit and spin-spin interactions, the special arrangement of the magnetic moments implies a corresponding ordering of the orientations of the orbital moments and spins relative to the crystalline lattice.

We now move on to the effect of doping. An important feature of Eqs. (6) is the global ferromagnetism along the $y$ axis. Weak as it is, the partial FM order in alternative $x z$ planes indicates that upon doping this weak FM component should be so enhanced that the FM phase arising at sufficient doping rates should also direct along this $y$ axis as observed experimentally. ${ }^{1,17}$ In other words, the PM to FM phase transition should also be associated with $\tau^{5}$. Formally, this is induced by the coupling of $L_{2 x}$ with $M_{y}$ in Eq. (4). Microscopically, the doped holes promote the mobility of the $e_{g}$ electrons that mediate the FM coupling. Accordingly, as the doping increases, the FM coupling is enhanced and hence $T_{c}$, the FM transition temperature, increases. On the other hand, doping suppresses the antiferromagnetism. Therefore, when the doping level $\delta$ (we use $\delta$ here instead of $x$ to avoid confusion) is not too large, we may assume that

$$
\begin{gathered}
a_{2}(\delta)=a_{0}\left(T-T_{N}+a_{0}^{\prime} \delta\right), \\
c(\delta)=c_{0}\left(T-T_{c}-c_{0}^{\prime} \delta\right),
\end{gathered}
$$

where $T_{N}$ and $T_{c}$ denote the AFM and the FM transition temperatures at $\delta=0$, respectively, and $a_{0}, a_{0}^{\prime}, c_{0}$, and $c_{0}^{\prime}$ are positive constants. Then, once $\delta>\delta_{c} \equiv\left(T_{N}-T_{c}\right) /\left(a_{0}^{\prime}\right.$ $-c_{0}^{\prime}$ ), the coefficient $c$ will become negative first upon cooling, and so the system exhibits a PM to FM instead of an AFM transition. In this case, a similar analysis yields a dominant magnetization $M \approx \sqrt{-c / d}$ with now a weak canting of $L_{2 x} \approx-\gamma_{7} M / a_{2}$ and $L_{3 z} \approx-\gamma_{9} M / a_{3}$ in contrast to the AFM state. This simple approximation is in qualitative agreement with the magnetic phase diagram at low doping as in Refs. 19 and 20; namely, the AFM transition temperature decreases but the FM one increases with increasing doping.

More importantly, this simple model for the competition between the two phase transitions exhibits phase separations at low doping. It is possible to extend the present theory to a generalized Ginzburg-Landau theory by including Coulomb repulsion and Boltzmann entropy terms for the holes as well as gradient contributions from spatial inhomogeneities to give a quantitative account. Here, to illustrate the essential point, it is instructive to compare the bulk free energy of a doped, uniform AFM state with that of a state composed of a hole-depleted AFM and a hole-rich FM phase. To this end, note that a uniform ordered AFM state at a doping level $\delta_{0}$ has a bulk free energy of $-a_{2}^{2} / 4 b_{2}$, neglecting the small relativistic contribution. Accordingly, when it is separated into, for instance, a pure AFM state with $\delta_{L}=0$ and another weaker AFM state of a number fraction $n\left(\delta_{0} \leqslant n<1\right)$ with a higher doping $\delta_{H}=\delta_{0} / n$ due to the conservation of holes, its bulk free energy alone is lowered by $(1-n) a_{0}^{2} a_{0}^{\prime 2} \delta_{0}^{2} / 4 b n$ $>0$. Meanwhile, the FM component also gains a bulk free energy of $n c^{2}\left(\delta_{H}\right) / 4 d$ or $(1-n) c_{0}^{2} c_{0}^{\prime 2} \delta_{0}^{2} / 4 d n$ for separation at high temperatures when $c\left(\delta_{0}\right)>0$ but $c\left(\delta_{H}\right)<0$ or at low temperatures when $c\left(\delta_{L}\right)<0$, respectively, while at intermediate temperatures, whether or not the FM component alone favors a separation depends on the system (via the parameters). These energies gained may possibly overtake the Coulomb energy cost for hole aggregation, particularly for the low doping levels at which the aggregated holes can still be distant enough to reduce their Coulomb repulsion. As a result, a doped system tends to separate into hole-rich regions with FM order and hole-depleted regions with AFM order. In reality, these electronically separated regions may be broken into microscopic pieces by the long-range Coulomb interaction in order to spread the charge uniformly. Furthermore, the FM and AFM orders may possess a certain variable strength depending on the concentration of doped holes owing to their common FM component. This accounts qualitatively for the coexistence of FM and AFM features ${ }^{1,9}$ and the 
liquidlike distribution of FM droplets observed in neutron scattering experiments at low doping. ${ }^{10}$

Note that the symmetry relationship between the two phases plays an essential role in the above analysis. The tendency to favor separation is caused by an instability in the inverse compressibility $\sim \partial^{2} F / \partial \delta^{2}=-\left(\partial a_{2} / \partial \delta\right)^{2} / 2 b_{2}$ $-a_{2}\left(\partial^{2} a_{2} / \partial \delta^{2}\right) / 2 b_{2}<0$ for an AFM state since doped holes always raise its energy by frustrating the AFM order. Similarly, the FM state favors more holes to a certain extent in order to lower the kinetic energies through double exchange. Nevertheless, for a single AFM or FM state, a phase separation is hardly possible because of the Coulomb repulsion for charged particles. In general, this fact is taken into account within Landau-Ginzburg theory by including quadratic terms to assure stability. In the present case, however, both the hole-depleted AFM phase and the hole-rich FM phase are energetically favored relative to a uniform state. An underlying mechanism that makes this separation feasible is the close symmetry correlation that facilitates the transition from the AFM state to the FM state by the itinerant holes. Thus, as the doped holes hop, they enhance the weak FM component of the AFM state via double-exchange interactions. Such enhanced FM regions can catch more holes which in turn can further strengthen the FM order. This avalanche effect due to a common FM component could trigger the separation of the holes into hole-depleted and hole-rich regions.

In conclusion, we have shown that both the PM to $A$-type AFM and the PM to FM transitions in undoped and moderately doped $\mathrm{LaMnO}_{3}$, respectively, correspond to the transition from a magnetic group Pnma 1' to $P n^{\prime} m a^{\prime}$, and are associated with the irreducible corepresentation $\tau^{5}$ of the parent phase. This irreducible representation allows an $A$-mode AFM, a $G$-mode AFM, and a FM order along the respective $a, c$, and $b$ axes in the Pnma setting. Accordingly, the $A$-type AFM phase of undoped $\mathrm{LaMnO}_{3}$ also possesses a FM component, albeit weak, which is identical in the direction with the FM phase present at moderate doping. This symmetry relation may lead to a phase separation into hole-depleted AFM regions and hole-rich FM regions in order to take energetic advantage of both phases. Such a competition of the two relevant phases may also work in other systems like $\mathrm{La}_{2-2 x} \mathrm{Sr}_{1+2 x} \mathrm{Mn}_{2} \mathrm{O}_{7}$ where phase separation was also observed, ${ }^{21}$ though their symmetry may be different. Nevertheless, further investigation is awaited.

This work was supported by the URC fund and a CRCG grant from the University of Hong Kong.
*Electronic address: zwang@hkucc.hku.hk

${ }^{1}$ E. O. Wollan and W. C. Koehler, Phys. Rev. 100, 545 (1955).

${ }^{2}$ R. M. Kusters et al., Physica B 155, 141 (1989); K. Chahara et al., Appl. Phys. Lett. 63, 1990 (1993); R. von Helmolt et al., Phys. Rev. Lett. 71, 2331 (1993); S. Jin et al., Science 264, 413 (1994).

${ }^{3}$ N. Furukawa, J. Phys. Soc. Jpn. 63, 3214 (1994); A. J. Millis, B. I. Shraiman, and R. Mueller, Phys. Rev. Lett. 77, 175 (1996); F. Zhong, J. M. Dong, and Z. D. Wang, Phys. Rev. B 58, 15310 (1998).

${ }^{4}$ Ion size mismatch is also possible: see, e.g., W. E. Pickett and D. J. Singh, Phys. Rev. B 53, 1146 (1996).

${ }^{5}$ C. Zener, Phys. Rev. 82, 403 (1951).

${ }^{6}$ A. Asamitsu et al., Nature (London) 373, 407 (1995); Y. Tomioka et al., Phys. Rev. Lett. 74, 5108 (1995); M. Imada, A. Fujimori, and Y. Tokura, Rev. Mod. Phys. 70, 1039 (1998), for a review.

${ }^{7}$ E. L. Nagaev, Phys. Lett. A 218, 367 (1996); S. Yunoki et al., Phys. Rev. Lett. 80, 845 (1998); S. Q. Shen and Z. D. Wang, Phys. Rev. B 58, R8877 (1998); S. Yunoki, A. Moreo, and E. Dagotto, Phys. Rev. Lett. 81, 5612 (1998); see also A. Moreo, S. Yunoki, and E. Dagotto, Science 283, 2034 (1999), for a review.

${ }^{8}$ P. G. Radaelli et al., Phys. Rev. Lett. 75, 4488 (1995); C. H. Chen and S.-W. Cheong, ibid. 76, 4042 (1996).

${ }^{9}$ G. Allodi et al., Phys. Rev. B 56, 6036 (1997).
${ }^{10}$ M. Hennion et al., Phys. Rev. Lett. 81, 1957 (1998).

${ }^{11}$ See, e.g., C. J. Bradley and A. P. Cracknell, The Mathematical Theory of Symmetry in Solids (Clarendon, Oxford, 1972).

${ }^{12}$ J. C. Tolédano and P. Tolédano, The Landau Theory of Phase Transitions (World Scientific, Singapore, 1987).

${ }^{13}$ O. V. Kovalev, in Representations of the Crystallographic Space Groups: Irreducible Representations, Induced Representations and Corepresentations, edited by H. T. Stokes and D. M. Hatch (Gordon and Breach, Yverson, 1993).

${ }^{14}$ E. F. Bertaut, Acta Crystallogr., Sect. A: Cryst. Phys., Diffr., Theor. Gen. Crystallogr. 24, 217 (1968).

${ }^{15}$ International Tables for Crystallography (Reidel, Boston, 1983), Vol. A.

${ }^{16}$ I. E. Dzyalashinskii, Zh. Eksp. Teor. Fiz. 46, 1420 (1964) [Sov. Phys. JETP 19, 960 (1964)].

${ }^{17}$ G. Matsumoto, J. Phys. Soc. Jpn. 29, 606 (1970); H. Kawano, R. Kajimoto, M. Kubota, and H. Yoshizawa, Phys. Rev. B 53, 2202 (1996).

${ }^{18}$ I. Solovyev, N. Hamada, and K. Terakura, Phys. Rev. Lett. 76, 4825 (1996).

${ }^{19}$ A. Urushibara et al., Phys. Rev. B 51, 14103 (1995).

${ }^{20}$ P. Schiffer, A. P. Ramirez, W. Bao, and S.-W. Cheong, Phys. Rev. Lett. 75, 3336 (1995).

${ }^{21}$ T. G. Perring, G. Aeppli, Y. Moritomo, and Y. Tokura, Phys. Rev. Lett. 78, 3197 (1997). 